\title{
THE SPIRAL-COMPACT GALAXY PAIR AM2208-251: COMPUTER SIMULATIONS VERSUS OBSERVATIONS
}

\author{
Mario Klarić \\ Department of Physics and Astronomy \\ University of Alabama \\ Tuscaloosa, Alabama 35487 \\ Gene G. Byrd \\ Department of Physics and Astronomy \\ University of Alabama \\ Tuscaloosa, Alabama 35487
}

\section{INTRODUCTION}

The system AM2208-251 is a roughly edge-on spiral extending east-west with a smaller round compact $\mathrm{E}$ system about 60 arcsec east of the spiral nucleus along the major axis of the spiral (Fig. 1a). Further details are in A Catalog of Southern Peculiar Galaxies and Associations (Arp and Madore 1987).

Bertola, Huchtmeier, and Zeilinger (1990) have presented optical spectroscopic as well as single dish $21 \mathrm{~cm}$ observations of this system. Their spectroscopic data show, via emission lines $\lambda \lambda 3727-29 \AA$, a rising rotation curve near the nucleus ( $\mathrm{A}$ in Fig. 1b). On the west half of the spiral's disk, the detectable curve does not extend farther than about 10 arcsec from the nucleus. Toward the east, the detectable curve extends all the way to the companion $(B, C)$ and past it (D). This is in accord with the visual photo, which shows the spiral more extended toward the companion. The rotation curve flattens to about 150 $\mathrm{km} / \mathrm{s}$ at about 7 arcsec from the nucleus $(B)$. Then, at $\approx$ 28 arcsec, it dips and then slowly rises as it approaches the companion (C). Near the companion, the curve abruptly reverses slope (D), ending at $\approx 67$ arcsec. The break in the curve $(B)$ is possibly due

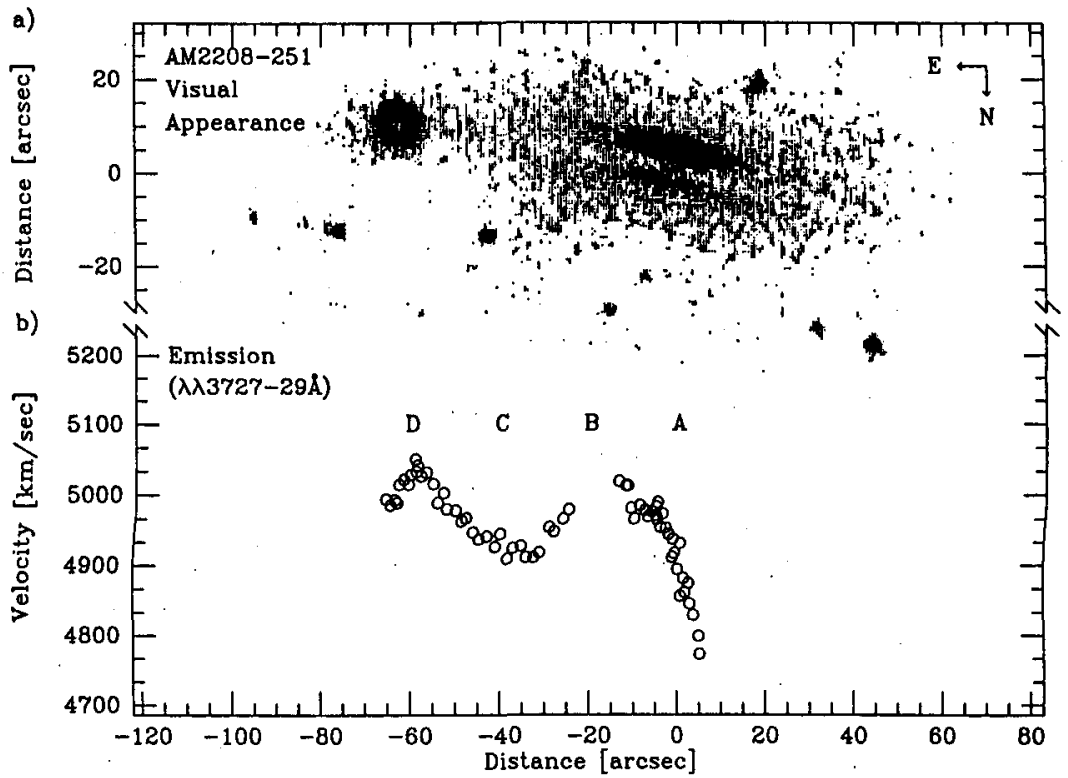

Fig. 1. a) Visual appearance of the system AM2208-251;

b) Velocities along the companion-spiral line. to absorption in the spiral's disk. These spectroscopic observations may indicate a tidal interaction in the system. In order to learn more about such pairs, we simulate the interaction using the computer model 
developed by Miller (1976 a,b, 1978 ) and modified by us (Byrd 1986, 1987, 1988). To do the simulation we need an idea of the mutual orbits of the two galaxies.

\section{ORBITS OF PAIR MEMBERS}

Since the E galaxy is compact, we can use the work in Byrd and Valtonen (1987) to infer that the $\mathrm{E}$ is a close companion in an orbit within roughly $30^{\circ}$ tilt with respect to the spiral's disk. The spectroscopic features indicate that the $\mathrm{E}$ galaxy has pulled gas from the spiral with the reversal in slope at D in Fig. 1b, interpreted as due to the E's gravitational field as the gas swings past the $E$ galaxy. If the companion is in a small inclination orbit with respect to the spiral's disk, the spectroscopic data indicate it is moving in the same sense (direct) as the spiral's disk turns. The radial velocity is positive with respect to the spiral's nucleus at the E galaxy's location, as is that of the part of the disk between the companion and the spiral's nucleus, so the two senses match.

A small orbit tilt is also consistent with the accretion of gas from the spiral by the companion. The edge of the disk turns to follow the companion as it swings past its perigalactic point. The velocity difference between the companion and disk edge of the spiral is reduced, increasing the ease of accretion (see Fig. 2 for computer simulation). Retrograde encounters have much higher velocities relative to the disk and do not affect the perturbed spiral as much (Byrd et al. 1986, 1987).

\section{THE COMPUTER MODEL}

Our computer model is a two-dimensional polar N-body program. It consists of a selfgravitating disk of particles, within an inert axially symetric stabilizing halo potential. The particles are distributed in a 24(radial) by 36(azimuthal) polar grid. Self consistent calculations can be done only within the grid area. The disk is modeled with a finite Mestel disk, where all the particles initially move in circular orbits with constant tangential velocities (Mestel 1963), resulting in a flat rotation curve. For more complete description of the program see Byrd et al $(1987,1988)$.

The gas particles in the spiral's disk, which make up $30 \%$ of its mass, collide in the following manner. The number of particles in each bin of the polar grid is counted every time step. If it is greater than a given critical density, all the particles in the bin "collide", obtaining in the result the same velocities - equal to the average for the bin. This process produces clumps of gas particles - the star formation sites. We suppress the collision in the inner part of our disk (within the circle $r=6$ ) to represent the "hole" seen in the gas in the nuclear bulge of spirals. We thus avoid spurious effects due to collisions in that region. We have also varied the size of the collisional bins, which did not affect our conclusions.

Under the guidance of the preceding reasoning and many different trials, we chose a direct encounter of a softened companion in a planar orbit. Using previous simulation experience, the degree of E-W asymmetry in the spiral, and the relative brightness of the companion and the spiral, we chose a companion 0.2 of the spiral galaxy mass which would approach to within 1.5 disk radii of the spiral on a zero energy direct orbit (roughly a parabola or elongated ellipse). With tidal energy loss the disturber actually gets to 1.36 disk radii. One disk radius corresponds to 36.44 arcsec, or $17.5 \mathrm{kpc}(H=50 \mathrm{~km} / \mathrm{s} / \mathrm{Mpc})$. Taking the disk orbital speed to be $150 \mathrm{~km} / \mathrm{s}$, one time step equals $1.17 \times 10^{6}$ years. 
The disturber is initially on the $\mathrm{x}$-axis in a "three-o-clock" position in Fig. 2a. This direction corresponds to the $0^{\circ}$ viewing angle by an observer, with viewing angle increasing counterclockwise in the Figures $2 a, 2 b$ and $2 c$. The disturber's initial tidal effects on the disk are minimal. It passes by the disk in a close approach, initially zero energy orbit affected by the halo field and the disk particles.

\section{RESULTS OF COMPUTER SIMULATIONS}

Which step in our "best choice" encounter orbit (Fig. 2a) best represents AM2208-251?

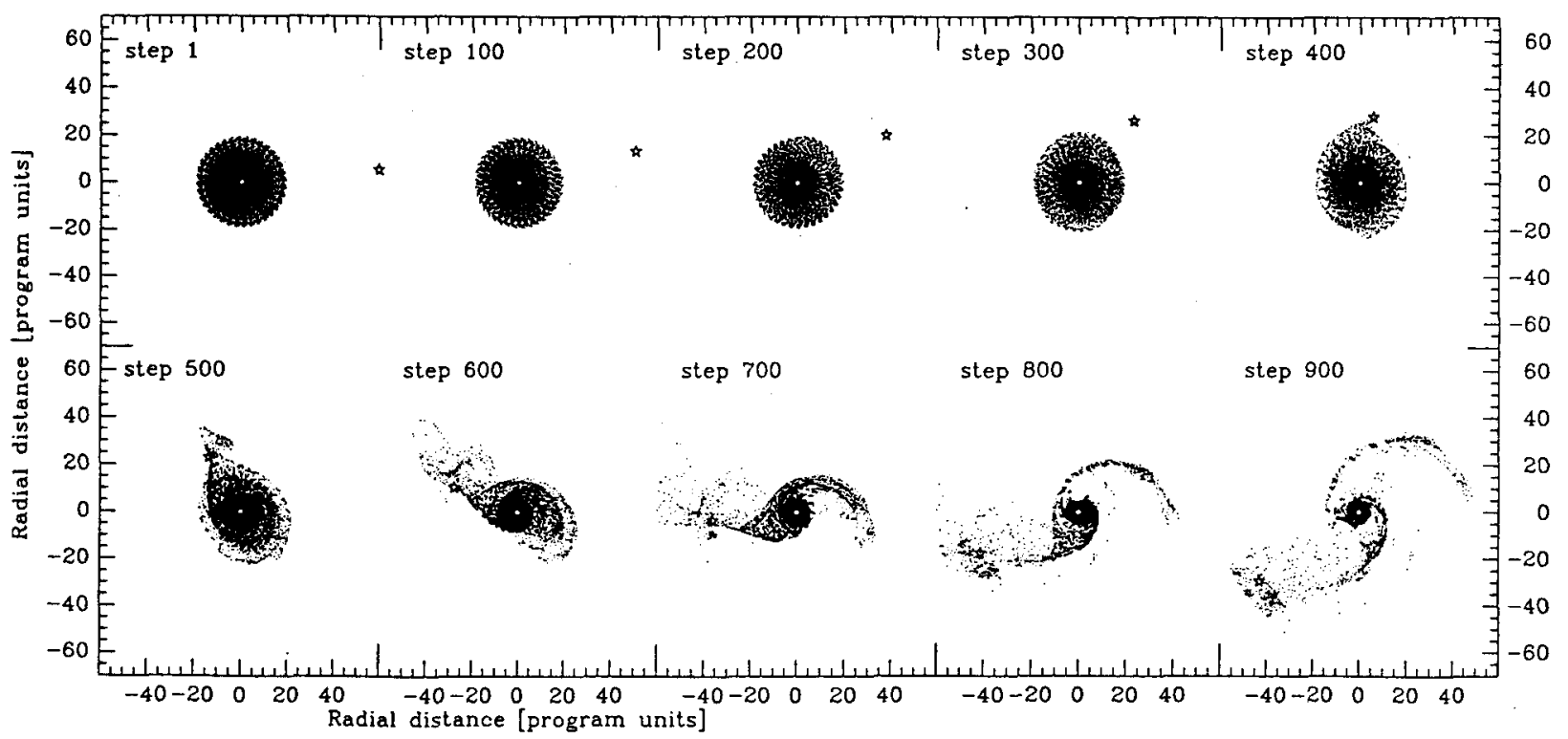

Fig. 2. Computer simulation results:

a) time steps 1 - 900;

b) time step 600 .

( 1 program unit $\approx 1.8$ arcsec)

We see time steps 1 through 900. A "bridge arm" starts to develop toward the companion around the step 400 , and is well developed at the step 500. The clumps start forming in the bridge arm. Around the step 700 the bridge starts to break, and the "far-side" arms on the other side of the disk are now more prominent. At the step 900 the bridge is broken, and the disturber is moving away from the disk. In light of this sequence above, the well-defined bridge in

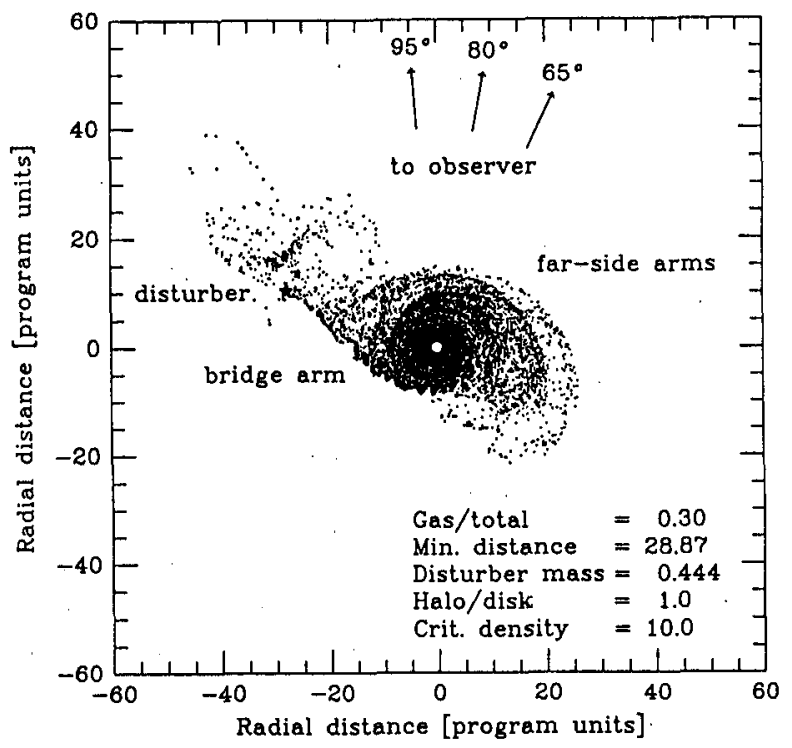
Fig. 1a implies that the companion has recently passed the perigalactic point. We display the result after perigalactic passage looking down from the galactic north pole on the disk in Fig. 2b. Examination of Fig. 1a indicates that, since the dust lane crosses the spiral nucleus slightly off-center (to the north), we are seeing the system in Fig. $2 \mathrm{~b}$ from the upper right side, slightly below the plane of the paper.

In our galaxy, and in other spirals, emission nebulae are most abundant in the densest parts of the spiral arms. Examining Fig. 2a, we see that our simulation produces a well- 
defined bridge arm with a narrow front edge extending out to the companion, and some arms on the opposite "far side". Step 600 (Fig. 2b) produces the best bridge arm with material swinging out past the companion. We assume the gas clouds have collided in the manner explained above, producing young stars and emission nebulae along the arms in the spiral. This is evident in Fig. 3, which shows a close-up of the Step 600 bridge arm, with velocity vectors of the gas particles shown. Since collisions of gas clouds are probably the mechanism for a massive star formation in arms, we compute vector average velocities for particles near one another in the arms. The collision products (merged clouds, young stars, and emission nebulae) will have this net, inelastic collision velocity. The vectors cross along the arms, where collisions would occur. Note the big clumps of collided gas cloud particles along the arms in Fig. 3. The particles in these clumps move parallel to one another as a result of collisions. Assuming that emission nebulae are concentrated along the arms in these clumps, we plot the radial velocities along those arms that would be seen by the observer.

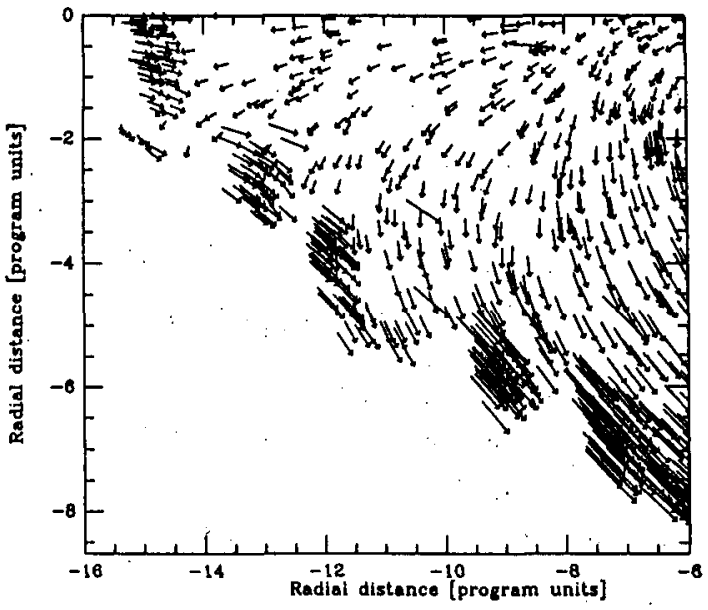

Fig. 3. The bridge-arm section of the time step 600 .

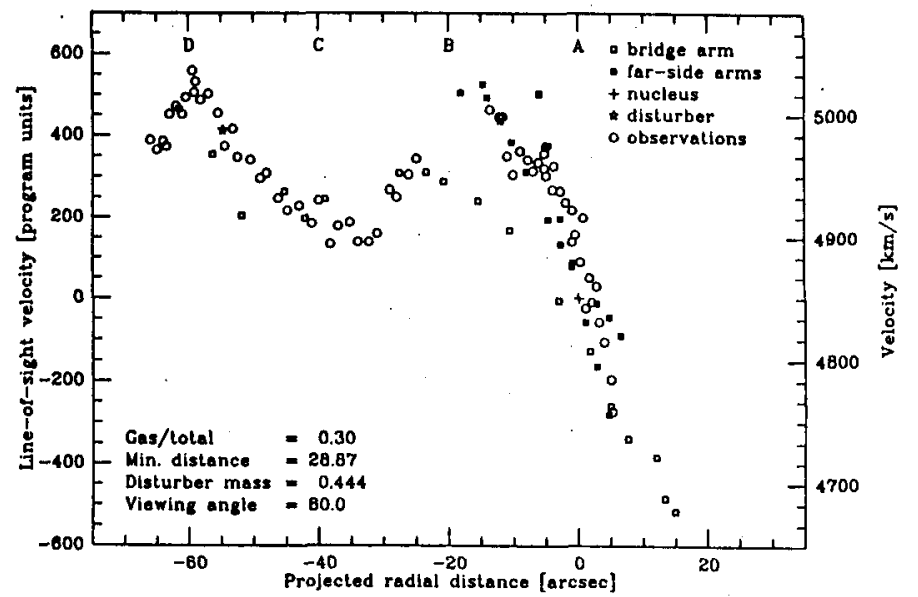

Fig. 4. The best-fit rotation curve with the observations.

Fig. 4 shows our model radial velocities versus position along the companion-spiral axis for step 600 (Fig. 2b) for the viewing angle of $80^{\circ}$ (the best fit), with the observations superposed. One can see the remarkable agreement between the two curves, and from that deduce what parts of the spiral are actually visible. We think that the part $A$ of the curve around the nucleus originates in the far-side arms, which are closer to the observer, and are blocking the bridge arm. The break in the observational curve (part B), from $\approx 14$ arcsec to $\approx 20$ east from the nucleus, is due to the absorption by the material in the spiral's disk.

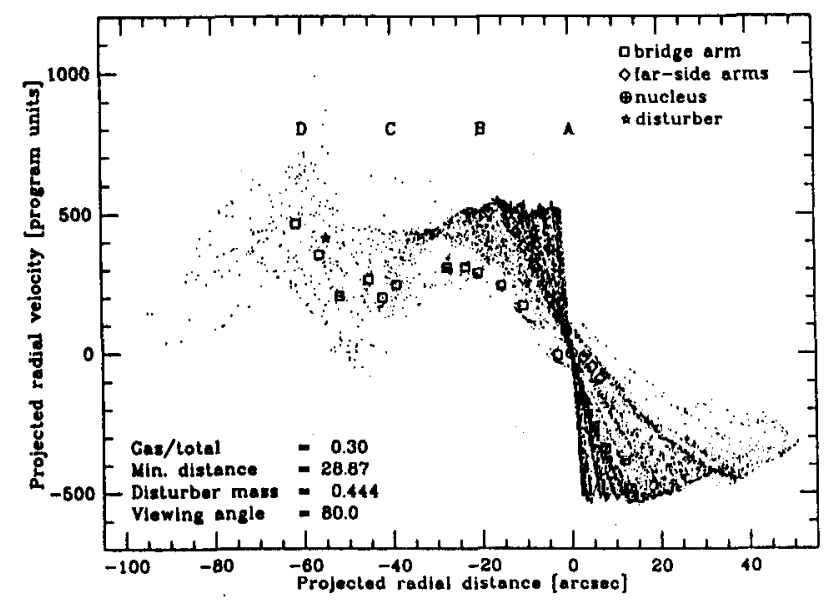

Fig. 5. HI velocity curve, with the best-fit rotation curve $\left(80^{\circ}\right.$ viewing angle). The bridge arm is visible from $\approx 20$ arcsec to $\approx 67$ arcsec east from the nucleus, with the dip, the rising slope (part C), agreeing very well 
with the observations.

Fig. 5 shows the radial velocities of the computer simulation emission nebulae (found along the arms) on top of all the gas particles in the disk. These clouds best represent atomic $\mathrm{H}$ or alternatively stars with strong absorption lines scattered over the whole disk. Here we have enough particles to see the reverse slope of the gas particles beyond the disturber (part D).

We have varied azimuthal viewing angle $\pm 20^{\circ}$ before the correspondence with observations breaks down. We have reasonable confidence in the planar orbit chosen, and in the mass of our companion based on its being a compact galaxy, and the relative brightness of the compact and the spiral (see the AM catalog). The halo/disk ratio is quite uncertain, but $1 / 1$ seems reasonable. The simulation results are not particularly sensitive to initial orbital energy. But the distance of closest approach (related to orbital angular momentum for a given energy) is crucial in forming the right sort of bridge. Beyond $\approx 1.5 \times$ that chosen (1.36 disk radii) no bridge forms; if the approach is $\approx 0.5$ that chosen, the disk is too greatly disturbed for the bridge arm to be stable.

Our results have implications in regard to possible measurement errors for rotation curves of nearly edge-on spirals. Recall Fig. 5 shows the optical HII regions rotation curve (squares) as well as the HI rotation curve (dots) in our computer simulation. In Fig. 5 note the great difference between the "real" HI tangent point rotation curve of our model galaxy $(\approx$ flat going from the nucleus outward) (left and right) and the rotation curves one would erroneously infer from the emission line measures of our edge-on model. Although one would certainly be cautious because of the spiral's edge-on orientation, our model results indicate that the gently rising parts of the emission line radial velocities near the nucleus in Fig. 1b are by no means a rotation curve. The actual curve is still flat close to the nucleus as the dots in Fig. 5 show. Also note that the curves for the far-side and bridge arms do not go through the nucleus' radial velocity. Again, the model points out how the observations may be misleading in disturbed spirals. Depending on the direction from which one observed this galaxy in HII, one could obtain almost exactly the nuclear velocity (the present side), or $\approx$ $100 \mathrm{~km} / \mathrm{s}$ off if we were looking from the opposite side.

\section{REFERENCES}

Arp, H.C. and Madore, B.F. (1987). A Catalogue of Southern Galaxies and Associations, Cambridge University Press

Bertola, F., Huchtmeier, W.K., and Zeilinger, W.W. (1990). in Proceedings of the Heidelberg Conference on the Dynamics and Interactions of Galaxies edited by $R$. Wielen. and

A. Toomre (in press) (Springer Verlag).

Byrd, G.G., Saarinen, S. and Valtonen, M.J. (1986). Mon.Not.Astron.Soc. 220, 619

Byrd, G.G., Sundelius, B. and Valtonen, M.J. (1987). Astron. Astrophys. 171, 16

Byrd, G.G., Valtonen, M.J., Sundelius, B. and Valtoaja, L. (1988). Astron. Astrophys. 166, 75

Mestel, L. (1963). M.N.R.A.S. 126, 553

Miller, R.H. (1976a). Journ. Comp. Phys. 21, 400

Miller, R.H. (1976b). Astrophys. J. 207, 408

Miller, R.H. (1978). Astrophys. J. 224, 32 